\title{
Labyrinthe
}

21 | 2005 (2)

Communauté en pièces : d'Europe, d'Islam et d'ailleurs

\section{Hors du commun}

Réflexions phénoménologiques sur la communauté

\section{Laurent Lavaud}

\section{(2) OpenEdition}

Journals

Édition électronique

URL : http://journals.openedition.org/labyrinthe/917

DOI : 10.4000/labyrinthe.917

ISSN : 1950-6031

Éditeur

Hermann

Édition imprimée

Date de publication : 22 juin 2005

Pagination : 121-140

Référence électronique

Laurent Lavaud, «Hors du commun », Labyrinthe [En ligne], 21 | 2005 (2), mis en ligne le 16 juillet 2008, consulté le 01 mai 2019. URL : http://journals.openedition.org/labyrinthe/917 ; DOI : 10.4000/ labyrinthe.917 


\section{HORS DU COMMUN \\ Réflexions phénoménologiques \\ sur la communauté}

Laurent LAVAUD

La communauté est entrée, comme la subjectivité avant elle, dans l'ère du soupçon. Derrière les identités ethniques, religieuses, culturelles, nationales ou étatiques, il faudrait débusquer une logique uniformisante et totalitaire, où le même triomphe au mépris de la différence, de la diversité et de la singularité individuelle. Pire: la communauté serait une machine à exclure, à fabriquer de l'étranger, du marginal ou de l'ennemi. Car inévitablement il y a un dedans et un dehors de la communauté, une appartenance et une exclusion: on en est ou on n'en est pas, sans échappatoire. Il conviendrait donc d'arracher la politique à cette logique binaire, à cette partition tranchée où l'être-ensemble se construit dans une indissoluble solidarité avec un être-contre, où l'identique et le commun ne prennent leur force et leur sens que d'avoir les vertèbres collées avec l'autre et le non-commun qu'ils rejettent.

On ne peut certes nier qu'il y ait en germe dans la communauté deux risques symétriquement inversés pour la vie politique : celui du totalitarisme et celui du communautarisme. Dans le totalitarisme, le triomphe d'une identité unique devient principe d'exclusion, voire d'extermination à l'égard de tous ceux qui ne partagent pas cette identité. Dans le communautarisme, c'est la prolifération des identités qui fait peser sur le corps social la menace d'un éclatement et d'une dissolution. Deux figures extrêmes et antithétiques de la dynamique communautaire donc: celle de la terreur que fait régner l'unique qui broie tout dehors et toute extériorité par rapport à lui ; celle de la dissémination, de la perte du pacte commun et la menace à terme de dissoudre l'égalité des citoyens. La communauté paraît donc par essence profondément ambiguë. Elle est à la racine de la démocratie, puisque tout pacte démocratique authentique suppose de mobiliser la totalité des citoyens, la communauté qu'ils forment, sans exclusive. Et en même temps, le 
mouvement profond de la communauté semble inévitablement conduire à la construction d'un dehors contre lequel on se prémunit, et à un repli sur une identité provisoirement rassurante, sur une pureté fantasmée en lesquelles se reconnaissent les seuls élus ou les seuls initiés. Toute communauté serait donc rongée de l'intérieur par sa propre logique, victime d'un destin inexorable qui conduirait ses membres à sacrifier à l'idolâtrie du même et au rejet de l'autre, de l'étranger.

Pour autant, faut-il radicalement se priver du secours de la communauté pour penser l'être-ensemble? Ou plus précisément, la notion de communauté a-t-elle en elle suffisamment de ressources pour déjouer le double mouvement d'identification interne et d'exclusion de l'autre qui sont à l'œuvre dans les figures que l'on vient d'évoquer?

Il me semble nécessaire pour répondre à ces questions de remonter à la racine de la communauté, et plus précisément à sa racine singulière, au cœur même de l'individu. Mon hypothèse est que si le mouvement communautaire semble bien souvent conduire à une impasse, c'est qu'il est considéré comme un donné, comme un déjà là ethnique, historique ou culturel, qui précéderait l'individu et lui dicterait sa loi, sa nécessité propre. Il n'y aurait pas au sens propre d'entrée dans une communauté, librement assumée comme un événement choisi, mais toute communauté prendrait ultimement la figure d'un destin initialement préinscrit dans l'être.

Or, la communauté n'est pas un destin qui surplomberait l'individu en le précédant, mais elle est un projet qui ne cesse de remettre en cause l'identité, de l'inquiéter, de brouiller les frontières établies, le partage entre le propre et l'étranger. La communauté ne doit pas être pensée comme ayant un appui exclusif sur le donné, l'antécédent ou le transmis, mais elle est toujours une survenue; elle ne cesse de s'inventer, de se construire ou de se reconstruire: en un mot, la communauté est toujours à venir.

Or, seule une enquête sur la genèse individuelle de la communauté permettra de mettre au jour cette dimension prospective de la communauté, cette ouverture fondamentale à l'altérité et à l'avenir. C'est bien dans l'individu libre que la communauté trouve son ouverture fondamentale vers une survenue, vers une radicale nouveauté. C'est encore l'individu qui est capable d'accueillir l'autre, l'étranger, de faire éclater les frontières du commun, de revivifier les identités, d'abolir les 
coutumes. Les ressources d'innovation, de création, d'altération du commun sont à puiser dans la liberté individuelle. Tournons-nous donc vers l'individu pour remonter aux sources de la communauté.

\section{Et moi, et moi, et moi...: la communauté intersubjective (Husserl)}

Cependant, de qui parlons-nous? Quelle figure donner à l'individu censé ouvrir la communauté à son autre, briser ses frontières, et conjurer le destin du repli identitaire? La figure la plus immédiate de l'individu semble être celle du sujet, celle de la conscience qui surplombe la modernité depuis Descartes. Le sujet conscient de lui-même, de ses pouvoirs et de sa puissance d'action, le sujet libre et volontaire: voilà l'individu dans sa forme originelle, dans son contour le plus net et le plus précis tel que l'a dessiné la tradition philosophique moderne. Le problème cependant - et il n'est pas mince - est que du sujet, précisément, on n'en sort pas. Une fois acquise la certitude de soi-même, une fois assurées la possession de la pensée et l'étreinte réflexive du cogito, la philosophie éprouve les plus graves difficultés à reconquérir l'extériorité, et plus exactement, à retrouver l'autre conscience, l'autre sujet. L'ombre du solipsisme menace toute entreprise philosophique qui remonte au sujet, à la conscience de soi, comme fondement ultime de la vérité. Dès lors, partir du sujet en cherchant en lui la racine fondatrice de la communauté, n'est-ce pas dès le départ une gageure, un pari impossible? Si le sujet s'empêtre pour ainsi dire en lui-même sans trouver d'issue véritable vers une extériorité concrète, comment serait-il capable de trouver en lui-même les conditions d'une communauté avec son autre, avec une autre conscience? Encore faut-il qu'il soit capable de rencontrer réellement autrui pour former avec lui une communauté.

Certes, les tentatives n'ont pas manqué dans l'histoire de la philosophie, et plus précisément dans l'histoire de la phénoménologie, pour conjurer ce danger du solipsisme. Si l'on suit le fil de la méditation inaugurée par Husserl, c'est par le biais de la notion d'intersubjectivité qu'un accès va s'ouvrir vers l'autre conscience. Alors que toutes les choses du monde sont intentionnellement constituées comme des objets pour ma conscience, autrui, en tant qu'il est un autre sujet, demeure inobjectivable: il n'est pas une chose du monde parmi d'autres; d'une 


\section{Labyrinthe, $n^{\circ} 21$}

certaine façon, pour Husserl, la relation avec autrui, précède le monde, elle est «une communion effective qui est précisément la condition transcendantale de l'existence d'un monde, d'un monde des choses et des hommes ${ }^{1} \gg$. Je ne rencontre pas l'autre dans le monde, puisque c'est par l'autre conscience qu'il y a un monde: le lien originel que ma conscience tisse avec la conscience d'autrui, ce que Husserl appelle «la communauté illimitée des monades » est première par rapport au monde objectif, à la nature commune, parce que c'est elle qui la rend possible.

Il y a cependant un paradoxe profond qui gouverne cette communauté intersubjective. Husserl souligne en effet qu'autrui ne peut m'apparaître que dans le champ de ma conscience: "C'est en moi que les autres se constituent en tant qu'autres ${ }^{2}$.» Le sujet reste premier par rapport à son autre: comme le souligne Paul Ricoeur, on est bien ici dans le cadre d'une «égologie décidée » où le primat du moi ne saurait être contesté ${ }^{3}$. Et cependant, incontestablement, autrui ne se constitue en moi que sur le mode d'une séparation, d'une radicale étrangeté, il résiste radicalement à toute véritable constitution objective. Je ne peux constituer autrui que sur le mode de l'inobjectivable, de ce qui ne doit jamais m'apparaître comme un objet du monde.

On comprend aisément pour quelles raisons Husserl maintient fermement cette priorité du sujet dans l'analyse de la relation à autrui. L'enjeu est pour lui d'assurer la possibilité de la rencontre de l'autre conscience, et ultimement, la possibilité d'une communauté véritable entre les sujets. Or, il est à ses yeux inconcevable de partir de la séparation, de la distance entre les consciences pour tenter désespérément de combler le gouffre initial qui s'ouvre entre elles, et poser ensuite comme «après coup» les fondements de cette communauté. Le seul terrain où la rencontre est possible est celui du moi. C'est de lui qu'il faut partir pour construire les conditions de la communauté:

En partant de moi, monade primitive dans l'ordre de la constitution, j'arrive aux monades qui sont autres pour moi, ou autres en qualité de sujets psycho-physiques. Cela implique que j'arrive aux autres non pas comme s'opposant à moi par leurs corps, et se rapportant, grâce à l'accouplement

\footnotetext{
1. Edmund Husserl, Méditations cartésiennes, V, trad. G. Pfeiffer et E. Levinas, Paris, Vrin, 1992, p. 209.

2. Ibidem, p. 208.
} 


\section{Hors du commun}

associatif et parce qu'ils ne peuvent m'être donnés que dans une certaine «orientation», à mon être psycho-physique [...]. Bien au contraire, le sens d'une communauté des hommes, le sens du terme «homme», qui, en tant qu'individu déjà, est essentiellement membre d'une société (ce qui s'étend aussi aux sociétés animales) implique une existence réciproque de l'un pour l'autre 4 .

La communauté concrète, historique des hommes, leur condition sociale, trouve sa racine transcendantale dans cette «existence réciproque» qui est une orientation fondamentale de la conscience. La communauté intersubjective, constituée intentionnellement dans la conscience anticipe donc la communauté sociale, la communauté humaine qui rassemble les individus que Husserl désigne ici du terme de «moi psycho-physique».

Cependant, soulignons-le, l'autre n'est ici atteint, dans cette orientation intersubjective fondamentale, que comme un autre sujet, ou autrement dit, comme un alter ego. L'autre n'est pas reconnu en tant qu'autre, mais bien en tant qu'identique à moi. De même que je constitue transcendantalement en moi l'ensemble de l'horizon communautaire, et ainsi que je constitue l'autre, l'étranger, comme un autre sujet, autrui est lui aussi reconnu comme une instance subjective qui me pose comme un autre ego: il y a réciprocité, et pour ainsi dire responsabilité partagée dans la constitution de la communauté intersubjective. Husserl parle en ce sens de «saisie analogisante» et de «transfert » du sujet vers son autre ${ }^{5}$. La difficulté vient alors de ce que la dynamique de l'identité, que l'on a déjà mise en avant lorsque l'on a évoqué les critiques modernes de la communauté, risque bien de recouvrir et d'engloutir la fondation intersubjective de la communauté. Je n'atteins de l'autre que ce qui est identique à moi, que ce qui en lui relève de la similitude ou de l'analogie avec ma propre position phénoménologique. Ou autrement dit, je dépouille autrui de son altérité en ne retenant de mon rapport à lui que ce qui le rend d'une certaine façon comparable à moi, assimilable à ma propre activité constituante. N'est-on donc pas fatalement conduit à rater autrui dès lors que le point de départ du

\footnotetext{
4. Edmund Husserl, op. cit., p. 209.

5. Ibidem, p. 180-181. Ces expressions renvoient plus précisément au rapport entre ma propre « chair » et celle d'autrui. Le concept de « chair » est essentiel pour appréhender la méditation husserlienne de l'intersubjectivité, mais je n'aurai pas la place de m'y attarder ici.
} 
mouvement qui nous conduit vers lui est la conscience subjective, cette «monade» dont parle Husserl, dont l'activité est par essence placée sous le signe du même? Et dès lors, et comme par ricochet, la fondation subjective de la communauté, cette communauté intersubjective transcendantale, censée assurer la condition de possibilité de la communauté historique et sociale n'enferme-t-elle pas cette dernière dans les rets de l'identique et de la similitude? On est loin, semble-til, de mon hypothèse initiale selon laquelle c'est l'individu qui, par sa dynamique propre, doit permettre à la communauté de se préserver des sortilèges de l'identité.

\section{Le monde commun (Heidegger)}

Suffit-il alors, pour donner une nouvelle chance à cette hypothèse, de modifier la figure de l'individu qui serait le pôle fondateur de la communauté, en substituant au sujet transcendantal husserlien la figure du Dasein heideggerien? Trouve-t-on dans le Dasein, l'existant humain, les ressources suffisantes pour assurer les conditions d'une communauté qui échappe à la tyrannie du même?

Certaines assertions de Heidegger dans Être et Temps semblent à cet égard très prometteuses. Fondamentalement, Heidegger refuse de partir d'une scission initiale entre un sujet fermé sur soi, «monadique» pour parler comme Husserl, et un monde extérieur qu'il s'agirait de rejoindre ou de reconquérir à partir de cette position initiale du moi. Et ce qui vaut pour le monde est vrai aussi du rapport à autrui : il n'y a pas deux sujets, deux tours d'ivoire qui se feraient face à face et qui chercheraient une issue pour se rencontrer, pour engager un dialogue. Le Dasein est dès l'origine «ouvert» à l'autre Dasein, il ne s'appartient pas d'abord à lui-même avant de se tourner vers autrui et de chercher à engager un mode de relation avec lui, mais il est tout aussi constitutivement et originairement tourné vers autrui que vers lui-même. Ce que Heidegger résume par cette formule : « l'être-là (Dasein) est essentiellement être-avec ${ }^{6} »$. À cet égard, certaines analyses d'Être et Temps concernant le phénomène de la solitude sont décisives. Lorsqu'il fait

6. Martin Heidegger, Etre et Temps, trad. F. Vezin, Paris, Gallimard, 1986, p. 162. 
l'épreuve de la solitude, l'être humain expérimente la carence de cet être-avec, de cette relation constitutive avec autrui. Isolé, le sujet est en manque d'autrui, il ressent un malaise ou un mal-être, ou tout au moins la solitude lui apparaît comme une privation. Que l'être humain ne se suffise pas à lui-même, qu'un isolement ou une solitude radicales fassent figure d'exception, montre bien que la relation à autrui est constitutivement inscrite dans l'être du Dasein.

Il ne faut donc pas selon Heidegger partir de l'ego, du sujet isolé et comme perdu en lui-même, pour penser le rapport avec autrui, ainsi que le voulait Husserl, qui sur ce point emboîtait le pas à Descartes. Le Dasein, l'être-là de Heidegger n'est précisément pas un sujet ou un ego dans la mesure où il ne se situe pas en retrait du monde ou d'autrui, et où il ne bâtit pas son identité et sa constitution propres dans cette distance et cette séparation. Et par conséquent, il est tout aussi fallacieux de penser autrui simplement comme un alter ego, comme un autre sujet qui me renverrait en miroir mon activité constitutive:

Les autres ne se rencontrent pas à partir du moment où l'on fait la différence préalable entre son propre sujet d'abord là-devant et les autres sujets qui se rencontrent eux aussi, ni en dirigeant un tout premier regard sur soimême pour commencer par y fixer le terme premier d'une différenciation. Ils se rencontrent à partir du monde dans lequel séjourne l'être-là7.

Il n'y a pas de connaissance de soi qui précéderait ou même qui s'opposerait à une connaissance d'autrui, mais je me connais dans la mesure où je connais autrui, et réciproquement. Il y a pour ainsi dire une fécondation mutuelle de la connaissance de moi et de la connaissance d'autrui. Ou autrement dit autrui m'est donné tout aussi originairement que je suis donné à moi-même. Or, encore une fois, cette donation n'a pas lieu à partir d'une conscience-source qui se projetterait analogiquement, mais les Dasein, les êtres humains se rencontrent «à partir du monde».

Ici apparaît une idée essentielle qui est celle de «monde commun» ou de «monde partagé». Heidegger ne cesse de le répéter, le Dasein que je suis n'est pas un ego immédiatement hétérogène au monde, abstrait par rapport à lui, mais il n'y a de monde que par le Dasein,

7. Ibidem, trad. F. Vezin modifiée, p. 161. 
que par l'activité quotidienne du Dasein. Ce que l'on peut traduire phénoménologiquement en affirmant que c'est le Dasein qui mondanéise le monde: toute la vie de l'homme, intellectuelle, affective, sociale peut être ressaisie comme ce processus de mondanéisation du monde. Il n'y a pas de monde déjà là, qui me préexisterait comme déjà constitué, mais c'est par mon existence active que le monde peut m'apparaître comme tel. Or, la grande idée de Heidegger en ce qui concerne le rapport à autrui est que ce dernier est rencontré comme un autre Dasein, c'est-à-dire qu'il constitue lui aussi une instance ouverte qui par son activité, son souci, participe de la mondanéisation du monde. Dès lors, rencontrer autrui, c'est le rencontrer comme le dit Hedegger, «au travail», c'est-à-dire avec l'ensemble des réseaux de sens, avec les rapports aux choses et aux autres étants qui constituent son existence propre, sa manière singulière de s'inscrire dans le monde. D'une certaine manière, lorsque je le rencontre, autrui amène son monde avec lui. Mais il se trouve que ce monde est aussi le mien. Heidegger nomme «coexistence» cette rencontre de mon monde et du monde d'autrui, et le fait qu'ultimement ces deux mondes forment un monde commun.

On atteint donc ici aux rives d'une première forme de communauté, qui est celle constituée par ce que Heidegger nomme la «sollicitude» (Fürsorge). Ici encore le rapport au monde est central pour expliciter le sens de cet existential. Éprouver de la sollicitude pour quelqu'un revient à être attentif à son rapport aux choses, à son insertion dans le monde: je me soucie d'un de mes proches par un service, par une attention, une écoute...Heidegger distingue d'ailleurs deux modes de sollicitude. Le premier, qui est la sollicitude substitutive, consiste à décharger l'autre du souci du monde : il s'agit d'accomplir une démarche à sa place, de résoudre un problème ou de mener une activité qui sont hors de sa portée. Le second est la sollicitude libératrice: il s'agit non plus de substituer à l'autre, mais de le rendre autonome, de l'affranchir des contraintes intérieures ou extérieures qui pèsent sur lui de sorte à le rendre capable d'assumer par lui-même en toute liberté le souci du monde. Ici par exemple le jeu de la parole et de l'écoute aura une importance décisive.

Ce qui frappe alors dans l'analyse heideggérienne de la sollicitude est que je ne me soucie d'autrui que dans l'horizon et pour ainsi dire sous l'emprise du monde qui nous entoure. La sollicitude pour autrui 
me renvoie immédiatement ou plus précisément nous renvoie immédiatement au monde qui nous est commun, que nous avons en partage: il s'agit toujours en dernière analyse soit de rendre l'homme capable de prendre en charge les choses qui l'entourent, les affaires qui le préoccupent, soit de l'alléger, de le décharger de ces soucis. En ce sens, si l'on durcit un peu l'analyse, il n'y pas réellement de communauté avec autrui, mais toute communauté se réduit à celle d'un monde commun (Mitwelt).

Je ne rencontre pas autrui directement, en lui-même, mais je le rencontre bien dans le monde, ou plutôt, comme le dit Heidegger «à partir du monde». Pour le dire autrement, le moment du face à face, de l'échange immédiat avec autrui est immédiatement envahi et recouvert par la préoccupation du monde : autrui me renvoie à mon insertion dans le monde, et, réciproquement, je renvoie autrui au souci du monde. Il s'agit donc en définitive moins de former une communauté que d'avoir quelque chose en commun, de partager quelque chose, qui est le souci du monde. Et ce qui est vrai de la communauté pourrait être dit d'autres attitudes existentiales qui impliquent l'être-avec-autrui, comme le fait de collaborer avec un autre, de travailler à un projet commun, ou d'entrer en compétition, d'être en lutte avec lui pour conquérir un marché ou pour obtenir une position. Ultimement, c'est toujours le monde qui est le lieu de la collaboration ou de la lutte, mais je peux à chaque fois passer à côté de l'autre, ne pas véritablement le rencontrer, ne pas le reconnaître dans un face à face immédiat où il m'apparaîtrait comme tel, une fois, pour ainsi dire, le monde mis entre parenthèses.

Une autre figure de la communauté apparaît cependant dans Être et Temps, qui accentue encore cette priorité du monde. Il s'agit de la «dictature du On». Dans la figure précédemment dégagée, le Dasein, qu'il soit à l'initiative de la sollicitude ou son destinataire, était appelé à assumer pleinement, en personne, son mode de rapport au monde et l'organisation de sens qui découle de sa position singulière au sein de celle-ci. Cette prise en charge personnelle du sens s'efface désormais dans le «on»: «Chacun est l'autre et personne n'est soi-même ${ }^{8}$ », souligne Heidegger. Le sens du monde est déjà là, il précède toute initiative de l'existant humain, et celui-ci ne peut que le subir ou le recevoir passivement. Dans le règne du «on», le monde d'une certaine

8. Ibidem, trad. F. Vezin modifiée, p. 171. 
manière se mondanéise sans moi, il s'organise et se structure, sans que j'aie à répondre en propre de son sens. Il y a d'ailleurs là une sorte de confort, de facilité pour l'être humain qui se laisse dicter passivement un sens déjà figé. Les exemples sont légion de cette domination du «on» dans l'existence quotidienne: il s'agit des règles sociales qui précèdent notre venue au monde, ou des phénomènes tels que la mode, la publicité ou l'opinion publique, phénomènes qui nous dispensent tous de prendre en personne des décisions de sens. D'une certaine façon, « on » pense et se comporte à notre place, il ne nous reste qu'à nous couler dans la dynamique collective et anonyme qui construit sans nous la signification du monde.

Dans cette nouvelle figure de la communauté, le monde reprend sa position de donné premier et antérieur qui serait « déjà là », avant même toute véritable initiative du Dasein:

La première chose qui soit donnée, c'est ce monde commun du «on », c'està-dire le monde avec lequel se confond le Dasein pour autant qu'il ne soit pas encore venu à lui-même, le monde dans lequel il peut toujours être de telle sorte qu'il ne soit pas obligé de venir à lui-même9?.

La communauté se fait ici à la fois anonyme, inapparente, et terriblement présente: elle règle les principaux aspects de notre vie quotidienne, les décisions les plus banales comme les plus essentielles, sans que l'on soit véritablement capable de désigner un sujet précis, déterminé, qui puisse répondre en propre de ces décisions.

On peut, semble-t-il, tirer des analyses de Heidegger une distinction nette entre deux figures concurrentes de la communauté. Dans la première, il s'agit de la communauté que je forme avec un «toi», des projets que nous faisons l'un avec l'autre, des rivalités qui nous unissent, des réseaux de sens que nous tissons ensemble, de notre insertion commune dans le monde, ou plus précisément de la manière dont ton monde et mon monde viennent à se juxtaposer et se confondre l'un avec l'autre, de sorte que nous partagions un monde commun. Dans la seconde, ce qui est en jeu est la communauté du tiers, de celui que je ne rencontre jamais comme tel, qui demeure anonyme, insaisissable,

9. Martin Heidegger, Prolegomena zur Geschichte der Zeitbegriff, GA, 20, Frankfurt, Klostermann, 1979, p. 339 (trad. J. Greisch pour ce passage, dans Ontologie et Temporalité, Esquisse d'une interprétation intégrale de Sein und Zeit, Paris, Puf, p. 167). 
qui est partout présent et qui partout fait défaut, qui s'impose malgré moi, qui habite secrètement bon nombre de mes décisions de sens. Dans la communauté du «toi», autrui est rencontré comme une personne unique, singulière, et qui répond d'une certaine façon à ma propre singularité. Dans la communauté du «on», autrui n'a plus de visage, il est pluriel, illimité, en aucune manière il ne me fait face. Et pour cause : le «on» est dans son essence la plus profonde une modalité du «soi », il pénètre, ou plus précisément il précède la constitution de mon identité personnelle.

\section{Le face à face avec autrui ou la communauté impossible (Levinas)}

La question qui se pose alors est la suivante: a-t-on véritablement épuisé la signification du rapport à autrui envisagé comme un «toi » dès lors que l'on situe cette signification sur le terrain du monde, du partage d'un souci commun vis-à-vis des choses qui nous entourent? Ou autrement dit, est-ce véritablement dans le monde, ou même à partir du monde que je rencontre autrui de la façon la plus originale et la plus authentique? C'est là précisément la question que pose la philosophie de Levinas à celle de Heidegger. L'expérience décisive ici est celle du visage d'autrui. Levinas distingue les phénomènes du monde qui font sens en tant qu'ils s'intègrent dans un réseau de relations, et l'apparaître du visage qui ne fait sens qu'à partir de lui-même, en étant d'une certaine façon séparé de toute relation, en portant une signification absolue qui ne repose en rien sur le rapport à l'autre que soi :

Le visage est signification et signification sans contexte. Je veux dire qu'autrui dans la rectitude de son visage, n'est pas un personnage dans un contexte. D'ordinaire on est un «personnage»: on est professeur à la Sorbonne, vice-président du Conseil d'État, fils d'untel, tout ce qui est dans le passeport, dans la manière de se vêtir, de se présenter. Et toute signification au sens habituel du terme est relative à un tel contexte: le sens de quelque chose tient dans sa relation à autre chose. Ici au contraire, le visage est sens à lui seul. Toi, c'est toi ${ }^{10}$.

10. Emmanuel Levinas, Éthique et Infini, Paris, Fayard, 1982, p. 90-91. 
Lorsque Levinas affirme que le visage m'apparaît sans contexte, coupé de toute relation à un être ou une chose, cela revient à dire que le visage d'autrui ne m'apparaît pas dans le monde ou à partir du monde. Le monde en effet n'est rien d'autre que le contexte lui-même envisagé dans son extension la plus large: il est ce réseau de significations qui se tisse à partir des relations infinies d'être à être, de chose à chose. Le visage, apparaître absolu, est hors monde, il ne prend sens pour moi à partir de rien d'autre que lui-même. En ce sens, Levinas affirme que le visage d'autrui me demeure définitivement incompréhensible: comprendre revient en effet à penser un phénomène en l'intégrant dans le monde, en le connectant aux êtres et aux choses qui peuvent lui donner un sens. Je ne peux m'emparer du visage d'autrui par un quelconque savoir ou une visée englobante. D'emblée, le visage d'autrui m'arrache au terrain de la connaissance pour me projeter dans l'ordre de l'éthique: il ne provoque pas un savoir, mais il m'assigne à une responsabilité.

Or, ce qui nous intéresse ici, est qu'autrui dans la relation éthique reste définitivement séparé, transcendant, par rapport à moi qui le découvre. Il y a une essentielle dissymétrie, une «hauteur» selon les termes de Levinas et une préséance d'autrui qui interdisent tout englobement dans une commune identité, toute abolition de la distance entre moi et le visage qui me fait face. Levinas ouvre la dernière section de Totalité et Infini par ces termes :

Le rapport avec autrui n'annule pas la séparation. Il ne surgit pas au sein d'une totalité et ne l'instaure pas en y intégrant Moi et l'Autre. La conjoncture du face à face ne présuppose pas davantage l'existence de vérités universelles où la subjectivité puisse s'absorber et qu'il suffirait de contempler pour que, Moi et l'Autre entrent en rapport de communion ${ }^{11}$.

La séparation par rapport à autrui est définitive: elle n'est pas une étape à surmonter, un moment négatif qui s'annulerait dialectiquement dans une communion plus haute. Autrui reste l'étranger qu'il s'agit d'accueillir, auquel il est de notre devoir d'offrir l'hospitalité: Levinas insiste fortement sur cette figure de l'hôte constitutive d'une nouvelle forme de subjectivité, qui ne s'accomplit pas dans le repli égoïste du sujet et dans sa clôture sur soi, mais qui est ouverture fondamentale à

11. Paris, Le Livre de Poche, p. 281. 
la souveraine irruption d'autrui. Dans cet accueil cependant, dans cette hospitalité que je lui offre, autrui reste autrui, il ne se départit en rien de sa hauteur et de son statut d'étranger. Accueillir autrui ne signifie en aucune façon le faire mien, ou même entrer en communion avec lui.

À ce point de notre parcours, la communauté s'avère donc impossible: le face à face avec autrui ne se laisse pas surmonter dans une unité supérieure ou intégrer dans une identité commune. Autrui est en tant que tel sans commune mesure avec moi, la dissymétrie entre lui et moi reste insurmontable: il ne s'agit pas ici de la rencontre de deux moi, de deux sujets qui se refléteraient l'un l'autre dans leur commune puissance, mais il s'agit d'un moi qui se trouve interpellé et d'une certaine façon convoqué par un toi. Le toi du visage n'a rien de commun ou d'identique par rapport à moi, il n'est absolument pas mon alter ego comme le voulait Husserl, ni même cet autre être humain avec lequel je partagerais un monde, ainsi que l'entendait Heidegger. On a désormais quitté le domaine de l'intersubjectivité, comme celui du monde commun. Hors du commun : voici la situation précise où se joue la relation avec autrui.

À partir de cette situation singulière, je ne suis pas d'une certaine façon figé, réifié ou fasciné par le visage d'autrui. Le face à face avec autrui engendre un certain nombre d'engagements possibles où mettre en jeu ma liberté: outre l'hospitalité que j'ai déjà évoquée, Levinas évoque le service, la bonté, l'amitié. Mais dans chacune de ces situations la distance avec l'autre n'est jamais abolie, l'ami reste le lointain, je ne lui rends pas service ou je ne fais pas preuve de bonté à son égard parce qu'il serait un autre moi-même (ce qui d'une certaine façon serait une sorte de calcul), mais parce qu'irréductiblement il est l'autre que moi, sans retour vers une illusoire identité.

\section{Une politique hors du commun (Derrida)}

Si l'on part alors de cette situation interpersonnelle où le moi reste irréductiblement séparé du toi, où il n'y a aucun fonds commun où élaborer une vie ensemble, comment peut-on encore poser la question de la politique ? A-t-on définitivement donné congé à toute forme de communauté? C'est précisément là le pari audacieux engagé par Jacques Derrida dans Politiques de l'amitié: penser la vie politique hors $d u$ 
commun, hors de toute forme de communion ou de toute communauté. Le terrain où s'engage la réflexion politique de Derrida est celui de l'amitié. Prenant le relais des médiations de Levinas et de Blanchot sur ce thème, Derrida part de l'idée que l'amitié transgresse toute forme de co-appartenance, d'identité culturelle, religieuse, ethnique, familiale ou politique. L'amitié est sans raison, elle ne suppose aucune condition préalable, aucun réquisit, aucune qualité particulière à laquelle devrait correspondre nécessairement l'ami. La question se pose alors: pourquoi suis-je l'ami de tel homme et pas de tel autre? Comment rendre raison de ce qui est sans raison, comment expliquer ce qui n'a pas de fondement? Pourquoi toute amitié est-elle singulière, élective, pourquoi ne suis-je pas l'ami de quiconque et de tout le monde, si en définitive rien ne permet de comprendre la relation que je tisse avec tel ami ? Voici la réponse, ou plutôt l'hypothèse avancée par Derrida:

Peut venir l'hypothèse que, s'il en est ainsi, c'est que l'amitié qui s'annonce dans ce langage, celle qui se promet ou promet sans rien promettre, elle n'est peut-être pas de l'ordre du commun ou de son contraire, de l'appartenance ou de la non-appartenance, du partage et du non-partage, de la proximité ou de la distance, du dedans ou du dehors, etc. Ni en un mot de la communauté ${ }^{12}$.

L'amitié, par sa dynamique au sens propre irrationnelle, par son absence de fondement, déjoue toute forme de communauté. Derrida tente alors - il est vrai avec une extrême prudence - d'esquisser une forme de politique dans l'espace ouvert par le caractère non communautaire de l'amitié :

Ce désir (...) qui, dans l'aimance - amitié ou amour - m'engage auprès de celui-ci ou de celle-ci plutôt que de quiconque, de tous et de toutes, auprès de ceux-ci ou de ceux-là (et non de tous et de toutes, et non de quiconque), auprès d'un «qui» singulier, fût-il en nombre, en nombre toujours petit, quel qu'il soit, au regard de «tous les autres», ce désir de l'appel à franchir la distance (nécessairement infranchissable) n'est (peut-être) plus de l'ordre du commun ou de la communauté, de la part prise ou donnée, de la participation ou du partage. [...] Et dès lors s'il y avait une politique de cette aimance, elle ne passerait plus par les motifs de la communauté, de

12. Jacques Derrida, Politiques de l'amitié, Paris, Galilée, 1994, p. 331. 


\section{Hors du commun}

l'appartenance ou du partage, de quelque signe qu'on les affecte. Affirmées, niées ou neutralisées, ces valeurs «communautaires» ou «communales » risquent toujours de faire revenir un frère. Peut-être faut-il prendre acte de ce risque pour que la question «qui? » ne se laisse plus politiquement arraisonner par le schème de l'être-commun ou en-commun, fût-il neutralisé, dans une question d'identité (individuelle, subjective, éthique, nationale, étatique, etc. $)^{13}$.

Le projet de Derrida consiste très précisément en une traduction politique de «cette courbure dissymétrique et de cette hétérogénéité infinie $^{14} \gg$ qui se jouent dans l'amitié. Ce projet politique dessine ce qu'il appelle une «démocratie à venir» où l'altérité de l'autre homme serait préservée, dans la distance et dans la séparation, sans pourtant que cette altérité puisse se laisser réduire à une forme de hiérarchie ou à un quelconque rapport de supériorité. L'autre pourrait donc se présenter à la fois sous la double détermination de l'égal et du dissymétrique, du non hiérarchique et de l'hétérogène.

\section{La communauté du tiers}

Est-il cependant simplement possible de tenir un tel pari d'une vie politique qui serait la traduction ou la prolongation de la dissymétrie essentielle à la relation interpersonnelle nouée dans la relation amicale ? L' ' à venir» de la démocratie ne risque-t-il pas ici de se laisser réduire à la pure et simple impossibilité du politique ? Ou encore, qu'est-ce qu'une vie politique qui a définitivement banni loin d'elle toute référence au commun, à l'identité sous quelque forme qu'elle prenne? N'est-ce pas donner pour point de départ à l'établissement du politique ce qui en constitue sinon la négation du moins ce qui y échappe définitivement, ce qui est radicalement hétérogène à l'ordre du politique ? Et en ce sens, une politique sans communauté, n'aboutit-elle pas à une politique sans politique?

Il me semble essentiel à ce point de la réflexion de clairement marquer l'écart entre deux ordres de relation à autrui, écart dont on

13. Ibidem, p. 331.

14. Ibidem, p. 259. 
avait d'ailleurs aperçu l'esquisse dans la pensée de Heidegger. Le premier ordre est celui du face à face, de la relation de personne à personne, du «moi» et du «toi». Ici, je rejoindrai les analyses de Levinas et de Derrida: la relation immédiate avec un toi se joue dans la distance et dans la dissymétrie. Le «toi» ne m'apparaît pas comme un autre «moi», ou tout au moins pas immédiatement. Ce n'est que dans un temps second, une fois relégué à l'arrière-plan l'événement de la rencontre, que je peux faire jouer la logique de l'identification, de l'alter ego: nous sommes sur le point de devenir amis parce que celui que je rencontre a les mêmes goûts que moi, parce que nous partageons des intérêts communs, ou que nous avons des connaissances communes, que nous avons vu les mêmes films ou les mêmes expositions... Mais fondamentalement, cette logique de l'identification, de la reconnaissance pourrait-on dire, n'est que l'écume immédiatement visible, mais aussi trompeuse, de ce qui est en jeu dans la relation d'amitié.

Ce qui reste essentiel chez l'ami, ce n'est justement pas ce qu'il partage avec moi, ce ne sont pas nos points communs, mais tout au contraire, ce qui me sépare de lui, ce qui fait qu'il demeure le lointain, l'étranger. L'amitié se nourrit de cette distance, de cet espace infranchissable. La relation interpersonnelle - qu'il s'agisse d'ailleurs de la relation amicale, ou d'une toute autre forme de relation, la lutte, la compétition, la coopération, l'entraide - se joue donc bien hors du commun. Par conséquent - et je me sépare de Derrida sur ce point -, il est impossible de traduire politiquement ce qui a lieu dans la relation de personne à personne. L'hétérogénéité demeure radicale, il ne saurait précisément pas y avoir d'espace commun où l'on puisse faire converger l'événement de la rencontre interpersonnelle et la logique propre au politique.

Car le politique ne peut pas se passer à mon sens de la référence à la communauté. Il y a à la racine du politique un essentiel partage, la postulation d'une identité, fût-elle universelle, et la constitution d'un projet ou d'un avenir commun. Le second ordre de relation à autrui, qui est l'ordre proprement politique, se dessine par conséquent non plus en référence à un toi qui me fait face, mais en lien, et, disons-le, en communauté avec un tiers que je ne rencontre pas immédiatement.

La difficulté est donc de penser une articulation possible entre l'ordre du «toi» et l'ordre du tiers, entre la relation dissymétrique de 
personne à personne, et la relation proprement politique à la communauté. Je l'ai dit, ces deux ordres sont hétérogènes; cela ne signifie pas qu'ils soient nécessairement indépendants, qu'il soit impossible de les articuler, d'établir une connexion de l'un avec l'autre. C'est là même le défi le plus essentiel que doit relever la pensée politique.

L'articulation entre l'ordre du «toi» et l'ordre du tiers doit être pensée de façon réversible: il n'y a pas de priorité de la communauté par rapport à la séparation propre à la relation interpersonnelle, ni de celle-ci sur celle-là. Mais chacun des deux ordres garantit d'une certaine façon à l'autre son espace de fonctionnement. En premier lieu donc, la connexion de la thématique de la vie politique à celle du face à face avec autrui garantit la communauté contre toute dérive totalitaire, tout sacrifice de l'individu ou de la personne au règne sans partage du même et de l'uniformité. Toute identification de la vie collective à un réseau de valeurs, à un système de références culturelles ou de dogmes ne cesse d'être inquiété et remis en question par l'événement de la rencontre singulière avec autrui. La pierre de touche qui permet d'éprouver le fonctionnement de la vie communautaire se résume à la question suivante: est-ce que l'ensemble des réseaux communs, des institutions, des valeurs identitaires propres à la vie politique viennent d'une certaine façon fausser la rencontre avec l'autre, peser sur elle et la dénaturer? Est-ce que le visage d'autrui s'efface sous la vague des réflexes identitaires, est-ce qu'il ne m'apparaît plus qu'à l'aune des références et des valeurs qui m'intègrent dans telle ou telle communauté ? Ou bien, au contraire, est-ce que l'événement de la rencontre transcende d'une certaine façon, et échappe dès l'origine à la logique communautaire, et déjoue radicalement tout clivage identitaire?

À ce point, l'autre, qu'il appartienne ou non à ma communauté familiale, religieuse, nationale, politique, excède toute tentative d'identification. Si cet excès n'est plus possible, si l'appartenance communautaire a subverti tout face à face, si j'en viens à faire le partage entre celui qui en est et celui qui n'en est pas, et si l'étranger ne m'apparaît pas dans toute relation où vient à moi une autre personne, mais se réduit en définitive à n'être que celui qui est hors de la communauté par opposition à celui qui est dedans, alors, assurément, la logique de la communauté dysfonctionne, elle est hypertrophiée, pathologique. Parce que l'autre, quel qu'il soit, demeure préservé comme l'étranger et le lointain, la relation singulière interpersonnelle est le rempart le plus fort 
contre toute forme d'exclusion: la séparation et la distance qui s'instaurent dans la rencontre d'autrui, qu'il appartienne ou non à la communauté, annule toute exclusion, toute frontière entre le dedans et le dehors de la communauté.

On en revient donc à l'idée que j'avançais au début de ces réflexions: c'est bien l'individu qui est le gardien du bon régime communautaire, c'est lui qui s'oppose à la tyrannie du même et de l'exclusion que peut secrètement recéler l'institution de la communauté. Encore faut-il maintenant préciser cette idée: ce n'est pas, on l'a vu, dans une quelconque fondation égologique de la communauté que l'on pourra trouver la garantie ultime aux dérives totalitaire ou communautariste. Ce n'est pas dans le moi en lui-même que la communauté trouve le facteur à la fois d'équilibre et d'ouverture qui contre la dynamique identifiante-excluante qui menace de l'envahir, mais dans l'événement premier de la rencontre avec autrui. C'est en définitive parce qu'autrui ne cesse de menacer mon identité, ne cesse de remettre en cause mes acquis, mes réflexes conservateurs, que je peux à mon tour ouvrir l'ordre politique, l'ordre commun vers l'innovation de l'avenir, vers l'irruption vivifiante de l'altérité.

Cependant, si la relation singulière au «toi », tant qu'elle est préservée dans sa puissance de subversion, d'altération, et de résistance au règne du même, garantit la communauté de toute clôture, de tout enfermement dans la logique identitaire, il faut aussi penser le mouvement inverse, celui qui va de la communauté à la relation interpersonnelle: en quoi l'ordre politique, l'ordre de la communauté, est-il nécessaire à l'équilibre de la relation du «moi» au «toi»? Pour le dire brièvement, j'avancerai l'idée que, paradoxalement, la communauté est ce qui garantit ma séparation d'avec autrui. Le risque qui se pose dans toute relation immédiate avec autrui qui me fait face, est très précisément de ne pas tenir la distance. Ainsi que je l'ai déjà évoqué plus haut, très vite, la dissymétrie dans la relation à autrui, le fait que par essence cette relation échappe au régime du même et de l'identité et aux exigences de la réciprocité risquent d'être enfouis, recouverts par toutes les procédures d'identification et de reconnaissance: je n'endure pas l'autre en tant qu'autre, mais je modalise ma relation avec autrui, j'atténue l'exigence que m'impose sa figure d'étranger, en décelant en lui ce qu'il peut partager avec moi, ce que nous pouvons avoir en commun. Dans la relation d'amitié, mais plus encore dans la relation amoureuse, la 
distance et la hauteur de l'autre courent à tout moment le risque d'être abolies et de disparaître - tout au moins fantasmatiquement - au profit d'un illusoire désir de fusion et de communion: retour donc de la logique du même et de l'identité, mais cette fois au cœur même de la relation singulière de personne à personne.

C'est à ce point que le tiers doit jouer sa fonction régulatrice. Le politique, l'exigence de la vie commune qui déborde de tout côté la stricte relation interpersonnelle, est un rempart contre la pulsion fusionnelle qui s'empare de l'ami ou de l'amant. La communauté du politique permet de prendre à rebours le fantasme de communion qui s'immisce dans la relation interpersonnelle. Les amants ne font pas qu'un parce qu'ils s'intègrent chacun individuellement dans un réseau social différencié, dans un contexte professionnel distinct, dans un champ politique propre. Le politique sépare donc les amants, comme les amis, il les rappelle à l'ordre du tiers, d'un vivre-ensemble qui fait éclater les frontières de la cellule interpersonnelle. Il y a donc bien ici une conjonction possible des logiques du tiers et du «toi » : la communauté maintient l'autre à distance parce que la situation sociale et politique de celui qui me fait face le fait d'une certaine façon échapper à mon emprise, à mon fantasme de communion. L'autre reste l'étranger, celui qui vient d'ailleurs parce que la communauté dans laquelle il s'insère l'arrache à ma puissance d'assimilation et de réduction au même.

Au terme du parcours, on est donc à même de souligner la distinction radicale des ordres, celui de la communauté et du politique d'un côté, celui de la relation éthique et interpersonnelle de l'autre. Mais les deux figures d'autrui ici mises en jeu, celle du tiers et celle du «toi», loin de se faire concurrence malgré leur hétérogénéité, se renforcent et s'affermissent l'une l'autre. La dissymétrie et la distance essentielles à la relation à l'autre personne cassent la logique uniformisante et assimilatrice qui habite toute constitution communautaire. Tout autre, dès lors que je le rencontre face à face, m'apparaît comme l'étranger, comme celui auquel je dois l'hospitalité: la frontière d'identification et d'exclusion, le dedans et le dehors de la communauté s'avèrent caducs, dépassés par la dynamique de la rencontre de l'autre personne. Et en retour, la communauté est ce qui maintient l'autre à distance: la présence anonyme du tiers ne cesse de troubler le face à face, elle réfrène le désir d'une impossible fusion, et fait échouer le fantasme 


\section{Labyrinthe, $n^{\circ} 21$}

d'une identité qui abolirait la séparation du «toi ». On est donc aux antipodes du soupçon, qui pesait sur la communauté politique, de servir la tyrannie totalisatrice du même et de l'identité : pensée dans son articulation avec la relation immédiate au «toi», la communauté est une instance de séparation, elle ne fait que creuser l'altérité de l'autre. 